\title{
"So wie zuhause“. Die private Sphäre als Arbeitsplatz polnischer Migrantinnen
}

\author{
DOBROCHNA KAŁWA
}

\section{Einführung}

Der folgende Beitrag widmet sich der Analyse der Migrationserfahrungen polnischer Frauen, die in deutschen Haushalten beschäftigt werden. Dabei stehen die Strategien im Fokus, mit deren Hilfe die Frauen die soziale und kulturelle Dissonanz zu minimieren versuchen. Diese Dissonanz ist ein Ergebnis der Einbettung der Frauen in zwei private Sphären in Deutschland und in Polen und zwei unterschiedliche Genderordnungen. Die Untersuchung ordnet sich in einen breiteren Kontext der Studien zur Migration der Frauen ein. Trotz der fortgeschrittenen Feminisierung der heutigen Migrationsprozesse befindet sich diese Thematik immer noch am Rande des Forschungsfelds.

Die hier präsentierten Ergebnisse wurden im Laufe einer qualitativen Studie gesammelt, die im Rahmen des bilateralen Forschungsprojekts „Grenzräume - Zwischenräume: Migrationsbewegungen von Polinnen ins Ruhrgebiet $^{\text {"1 }}$ durchgeführt wurde. Eines der Ziele des Projekts war, die Migrationserfahrungen der Frauen, die im häuslichen Sektor arbeiten, unter dem Aspekt des Austauschs der kulturellen Muster zu analysieren. Dieser Beitrag bezieht sich auf Interviews, die 2005 und 2006 mit Frauen durchgeführt wurden, die zu dem Zeitpunkt der Untersuchung in Deutschland arbeiteten und während ihres temporären Aufenthalts in Polen befragt werden konnten.

In dem Beitrag werden zuerst die gesellschaftlichen und historischen Quellen des heutigen Migrationsverhaltens der Polinnen dargestellt. Hier spielen die Aspekte eine primäre Rolle, die mit der Erwerbstätigkeit der Frauen verbunden sind. Die makro-soziologischen Aspekte bilden eine Einführung

1 Das Projekt wird von Prof. Sigrid Metz-Göckel geleitet, von der Volkswagenstiftung finanziert und an den Universitäten in Dortmund und Krakau realisiert. 
in den zweiten Teil des Beitrags, in dem ich die Untersuchungsgruppe und den theoretischen Hintergrund der Analyse präsentiere. Die eigentliche Analyse fokussiert sich auf die Übertragung der einheimischen kulturellen Normen und Muster in der privaten Sphäre auf die Arbeit in den deutschen Haushalten. Die Analyse der von den Migrantinnen benutzten Adaptationsstrategien führt zur Reflexion über die mehrdimensionalen Folgen dieses Verhaltens. Einerseits ermöglichen sie, das Gefühl der sozialen Degradierung, das sich auf den niedrigen beruflichen Status der Migrantinnen bezieht, zu minimieren; andererseits führen sie jedoch zu der Festigung der konservativen Genderordnung, in der die Frau der privaten Sphäre zugeordnet bleibt.

\section{Frauen in der Migrationsforschung}

In der polnischen Migrationsforschung erscheint die Geschlechtsunterscheidung, wenn überhaupt, nur als eine statistische Makro-Kategorie, die mit dem kulturellen Geschlecht nichts zu tun hat. Die Bedeutung des Geschlechts wird in Bezug auf die Push- und Pull-Faktoren der Migration, die Formen der ausgeübten Arbeit, die Dynamik der internationalen Mobilität, die Adaptationsstrategien am Aufenthaltsort und die eingebetteten Wertesysteme vernachlässigt. Die klare Unterscheidung der Migranten nach Geschlecht (Gender) und nach den von ihnen angenommenen gesellschaftlichen Rollen erscheint erst in den Migrationsanalysen aus der Mikro- oder Familienperspektive. Die Tendenz, die Migration der Frauen vor allem im familiären und häuslichen Kontext zu unterscheiden, womit sie im Rahmen der etablierten Migrationsforschung marginalisiert wird, ist ein Ergebnis der „Geschlechtsblindheit“ der Migrationsforscher (Harzig 2006). Dafür gibt es mehrere Gründe: Zuerst wird Migration in der Forschung als ein Phänomen betrachtet, in dem die Männer die Hauptrolle spielen, da sie arbeiten und Geld verdienen und an dem die Frauen nur begrenzt beteiligt werden, vor allem als Mitglieder einer Familie, der sie sich im Ausland anschließen (Kofman 1999). Zweitens berücksichtigen die bekanntesten theoretischen Ansätze keine kulturelle Geschlechtsunterscheidung (Tacoli 1999). Die neuen ökonomischen Ansätze unterstreichen zwar die individuellen Aspekte der Migrationsentscheidung oder die Bedeutung der Familie und des Haushalts, sie fokussieren sich aber auf die männliche Motivation zur Migration (De Jong/Richer/Isarabhakdi 1996). Drittens können die in privaten Haushalten arbeitenden Migrantinnen in quantitativen Studien nur schwer erfasst werden. Erst die qualitativen Methoden erlauben, das Problem der „Geschlechterblindheit“ zu überwinden und die Besonderheiten der Migration von Frauen, die Handlungsmuster, Adaptationsstrategien und die Identitätsbildungsprozesse zu untersuchen (Borkert et al. 2006). 
In Bezug auf polnische Studien haben wir noch mit einem weiteren Grund der Marginalisierung von Frauen zu tun, nämlich dem Fakt, dass erst seit kurzem die feministischen Theorien und das Thema des Genders in den Sozialwissenschaften präsent sind. Migration von Frauen gehört $\mathrm{zu}$ den neuesten Bereichen der Sozialforschung in Polen, deren Erscheinen - neben den theoretischen Gründen - mit den neuen Migrationsströmen aus Polen nach 1989, die einen hohen (70\%) Anteil von Frauen aufwiesen, verbunden ist. Die Studien zur Stellung der Migrantinnen in privaten Haushalten in Belgien, Italien, Griechenland, Spanien und Deutschland haben eine Reihe von wichtigen Erkenntnissen zu der Besonderheit der Frauenmigration gebracht (GrzymałaKazłowska 2001b; Romaniszyn 2003; Kępińska 2005; Kuźma 2005; Rosińska-Kordasiewicz 2005; Slany 2005; Zamojski 2005).

\section{Die Frauenpolitik Polens und die Stellung der Frauen auf dem Arbeitsmarkt}

Polnische ForscherInnen verwiesen auf die Push-Faktoren in den Aufnahmeländern als einen der Gründe für die heutigen Veränderungen der Frauenmigration. In diesen Ländern kommt es zur doppelten Segmentierung des Arbeitsmarks: einerseits werden gut bezahlte, anerkannte und hoch qualifizierte Arbeitsstellen durch Einheimische und die unqualifizierten und schlecht bezahlten durch Migranten besetzt. Andererseits wird zwischen typisch männlichen (z.B. Bauarbeiten, saisonale landwirtschaftliche Arbeit) und typisch weiblichen (in Haushalten) Tätigkeiten unterschieden (Lukowski 2004). Der Geschlechtsdualismus wird sowohl von den Arbeitgebern, die bestimmte Arbeitsplätze zur Verfügung für Frauen oder Männer stellen und sie entsprechend rekrutieren, als auch von den Arbeitnehmern, die sich auf bestimmte Stellen bewerben, praktiziert und reproduziert. Ein Beispiel dafür ist die Feminisierung von Haushaltsdienstleistungen, die überwiegend für und von Migrantinnen angeboten werden. Die Herausbildung dieser Migrationsnische und die Einstellung von fast ausschließlich Frauen weist auf die Dauerhaftigkeit der kulturellen Normen und der Definition der haushaltsverbundenen Tätigkeiten im Herkunfts- und Aufnahmeland als Domäne der Frauen. Die in Haushalten arbeitenden Migrantinnen übernehmen die Aufgaben ihrer Arbeitgeberinnen, die erwerbstätig sind und ihre traditionelle soziale Rolle nicht erfüllen (wollen), aber sich gleichzeitig eine solche „Vertretung“ finanziell leisten können. Die wachsende Nachfrage nach Betreuungsdiensten hängt auch mit der Alterung der deutschen Gesellschaft und der Struktur und den hohen Kosten von Pflegedienstleistungen zusammen, die die Einstellung von zeitlich flexiblen und billigeren Migrantinnen begünstigt. Andererseits erlaubt die Arbeit in Haushalten den Frauen die Nutzung ihres kulturellen 
Kapitals - der Fähigkeiten und Kenntnisse, die - laut der Informantinnen auch im Aufnahmeland benötigt werden. Zu den häufig erwähnten Fähigkeiten gehören die effektive Arbeitsorganisation, Kochkünste, Planung eines Tags für sich und den Schützling und das Verhalten gegenüber Ärzten, professionellem Pflegepersonal oder Krankenschwestern. Anderseits, was die spätere Analyse zeigt, dient das kulturelle Kapital den Migrantinnen, die eigene Stellung gegenüber den Arbeitgebern und Schützlingen zu interpretieren.

Seltener werden die Bedingungen im Herkunftsland analysiert, die die Arbeitsmigration von Frauen ebenfalls stimulierten - vor allem die strukturellen Veränderungen nach 1989 auf dem Arbeitsmarkt und damit auch in der Ordnung der Geschlechter. Die ersten Jahre der Transformation brachten eine zunehmende Diskriminierung von Frauen auf dem Arbeitsmarkt in Polen. Dies zeigte sich zum Beispiel in der niedrigeren Vergütung von Frauen, die zwischen 79-91\% der Vergütung von Männern lag. Für die postkommunistischen Länder war auch der plötzliche und deutliche Rückgang der Zahl der erwerbstätigen Frauen charakteristisch (Fábián 2005). In Polen ist die Erwerbstätigkeitsrate $^{2}$ von Frauen in den 1990ern von 57\% im Jahr 1988 auf 38.4\% im Jahr 2002 gesunken (GUS 2003b). Der größte Rückgang, um 63\% seit Anfang der 1990er, wurde in der Gruppe von Frauen über 55 festgestellt, wozu die eingeführte Möglichkeit der vorzeitigen Pensionierung führte. ${ }^{3}$ Das verkürzte Arbeitsalter und die niedrigere Vergütung zeigten sich in den niedrigeren Renten, die um 79\% der Renten von Männern liegen (Wóycicka 2004). Ferner wurden die Frauen von der dramatisch (von $0.3 \%$ im 1. Quartal 1990 auf 14.3\% im Dezember 1992) wachsenden Arbeitslosigkeit in viel höherem Ausmaß getroffen als Männer. Die Arbeitslosenquote ${ }^{4}$ der Frauen betrug 2003 20\% und bei Männern 18\%. Frauen waren auch öfter als Männer von Langzeitarbeitslosigkeit betroffen (Balcerzak-Paradowska 2004). Anders als bei Männern betraf die Arbeitslosigkeit auch hoch qualifizierte Frauen. Heutzutage bilden Frauen im Alter von 25 bis 34 Jahren, die auf dem Arbeitsmarkt auf Grund ihrer Rolle als Mütter benachteiligt werden, die größte Gruppe der Arbeitslosen. Arbeitgeber vermeiden die Einstellung von Frauen im Reproduktivalter aus der Überzeugung, dass die Kosten für Arbeit von Personen (Frauen), die familiäre Verpflichtungen haben, höher sind und dass

2 Erwerbstätigkeitsrate ist in Prozent das Verhältnis der berufstätigen Personen zu allen Personen über 15 Jahren.

3 Im polnischen Recht beträgt das Rentenalter für Frauen 60 Jahre und für Männer 65. Das Recht auf vorzeitige Pensionierung haben Frauen über 55 mit einer mindestens 30-jährigen Arbeitszeit und Männer über 60, die 25 Jahre lang gearbeitet haben und vollständig arbeitsunfähig sind. Andere Regelungen gelten für LehrerInnen unabhängig von ihrem Geschlecht, die nach 20 Jahren Lehrtätigkeit mit 55 pensioniert werden können.

4 Zahlen für 2003, von allen Frauen über 15 Jahren. 
ein Mutterschutz- oder Erziehungsurlaub zu organisatorischen Problemen in der Firma führt. Eine weitere Hürde bildet das Recht, das dem Arbeitgeber verbietet, einen Arbeitsvertrag während der Schwangerschaft einer Frau aufzulösen. In der Folge beeinflussen solche Kriterien wie Familienstand oder familiäre Situation die Erwerbstätigkeit der Frauen. In Vorstellungsgesprächen werden die Bewerberinnen nach ihren Kinderwünschen gefragt, manchmal verlangen die Arbeitgeber ein Arztattest, das bestätigt, dass die Bewerberin nicht schwanger ist (Hebda-Czaplicka/Kołaczek 2001). Die Probleme beziehen sich auch auf die graue Zone des Arbeitsmarkts, wo Frauen 2004 485.000 (37\%) aller Beschäftigten bildeten. Sie arbeiten meist in feminisierten Sektoren wie medizinische Pflege, Krankenpflege, Körperpflege, Kinderbetreuung und Haushaltsdienste. Die meisten Frauen entscheiden sich für eine illegale Beschäftigung, weil sie, ähnlich wie die Männer, war eine legale Arbeit aber ein zu niedriges Einkommen haben. Anders wie bei Männern arbeiten Frauen oft nur einen Monat lang (GUS 2005).

Aus der historischen Perspektive ist es bemerkenswert, dass die Aktivierung der Frauen auf dem polnischen Arbeitsmarkt in der kommunistischen Periode ein Ergebnis ambivalenter Politik des Staates war, die die Emanzipation der Frauen im Berufsleben, aber nicht die notwendige Veränderung der Rolle der Frauen in der privaten Sphäre unterstützt hat. In der Folge wurden Frauen doppelt belastet, mit beruflichen und häuslichen Verpflichtungen. Die Beschäftigungspolitik strebte nach Abschaffung der Arbeitslosigkeit, wobei die Löhne auf niedrigem Niveau blieben, so dass eine Familie mit einem einzigen Einkommen an der Grenze zur Armut lebte. Die Aktivierungspolitik wurde auch mit härteren Methoden durchgesetzt, z.B. war ein Kindergartenplatz in den 1950ern von der Beschäftigung der Mutter abhängig. In den folgenden Dekaden war der ökonomische Zwang ein effektiver Mechanismus der Kontrolle des Arbeitsmarkts. In den 1970ern kam es zu einem Kurswechsel. Die neue Politik fokussierte sich auf die Begrenzung der Beteiligung von Frauen am Arbeitsmarkt. Damals wurde der bezahlte Mutterschaftsurlaub eingeführt, wodurch Frauen, die eine finanzielle Gratifikation bekamen, sich aus dem Berufsleben für einige Zeit zurückzogen und die, die vor allem in niedrig bezahlten Berufen tätig waren, nach dem Urlaub weiter zu Hause blieben (Kurzynowski 2000). In dieser spezifischen gesellschaftlichen und politischen Situation verlor die Arbeit der Frauen ihren Status als Mittel der Emanzipation und wurde von den Frauen eher als eine Form des ökonomischen Zwangs oder doppelter Belastung betrachtet. Trotzdem verbreitet sich das Bild der berufstätigen Frau, was man auch daran erkennen kann, dass die Zahl der Arbeitsemigrantinnen wächst, die ihre Pflicht, die Familie mit zu unterstützen, wahrnehmen.

Aus ökonomischer Sicht wird die Migration von Frauen sowohl durch die komplementäre Situation auf den Arbeitsmärkten des Herkunfts- und Auf- 
nahmelands als auch durch die ähnliche Betrachtung der Rolle der Frauen in beiden Ländern ${ }^{5}$ stimuliert. Die dynamische Entwicklung der Migration von Frauen hat ihre nicht-ökonomische Wurzel vor allem im gesellschaftlichen Wandel nach 1989, der ein neues Trauma verursacht hat. Unter diesen Bedingungen wandern Frauen aus, weil die Migration die einzige Option für sie ist, die ihnen entweder die Selbstentfaltung oder die Erfüllung der Lebensziele der ganzen Familie ermöglicht. Im letzten Fall haben wir es mit der für die globale Migration von Frauen typischen Eigenschaft zu tun, die eine kollektive Strategie des ganzen Haushalts und nicht nur der Frau ist. Die Neigung von Frauen zur Migration wächst bei ledigen Frauen oder wenn im Haushalt mehrere Personen, aber weniger pflegebedürftige Kinder leben. Die Pflichten der Frauen werden dann von den anderen Haushaltsmitgliedern übernommen, obwohl es auf Grund der bisherigen Studien schwierig ist festzuhalten, wie die eigentliche Aufteilung der Aufgaben aussieht und inwieweit die Migration zur Redefinition der Geschlechterordnung führt (Kępińska 2005).

Das bisherige Wissen über die Migration von polnischen Frauen basiert vor allem auf allgemeinen Studien auf der Makro-Ebene und der qualitativen Analyse der Mobilität von Frauen, die ihre beruflichen Qualifikationen nach 1989 erworben haben. Im Falle von Migration nach Deutschland beobachten die Forscher sowohl bei Frauen als auch bei Männern eine Zunahme von PedelmigrantInnen, wobei Frauen öfters in Haushalten arbeiten und Aufgaben wie Reinigung, Kinderbetreuung oder Pflege von älteren oder behinderten Personen übernehmen. Für diese Migration ist spezifisch, dass die Migrantinnen in der privaten Sphäre arbeiten. Diese private Sphäre - nach einer weit gefassten Definition - betrifft nicht nur den häuslichen Raum, sondern auch die Freizeit und interpersonelle Beziehungen (mit den Arbeitgebern und mit dem Schützling), also die intimsten Bereiche (Klich-Kluczewska 2005). In

5 Informationen darüber liefern z.B. Befragungen zur Situation von Frauen und Familien in Europa aus dem Jahr 1994. Die Analyse der Ergebnisse weist auf bestimmte Ähnlichkeiten der kulturellen Geschlechtsmuster in Polen und Deutschland hin (nur alte Bundesländer). In beiden Ländern wird am meisten akzeptiert, wenn Frauen, deren Kinder zu Hause sind (gehen nicht in den Kindergarten oder zur Schule), sich um den Haushalt kümmern (Deutschland 62\%, Polen 68\%). Nur 1\% der Befragten in Deutschland meinten, dass in dieser Situation Frauen berufstätig sein sollten. In Polen waren es 10\%, wobei Teilzeitarbeit $27 \%$ der Deutschen und nur 13\% der Polen zustimmen würden. Interessant ist ein weiterer Vergleich: 36\% der Deutschen und 48\% der Polen glauben, dass ein allein stehendes Elternteil genauso so gut Kinder erziehen kann als beide Eltern zusammen. Allerdings meinen 59\% der Polen und nur 15\% der Deutschen, dass sich für das Wohlbefinden der Kinder die Eltern nicht scheiden lassen sollten. Mangelnde Akzeptanz von Ehescheidungen hängt mit dem Einfluss der Katholischen Kirche zusammen, auch in Italien, Irland und Spanien (Cichomski/ Jerzyński/Zieliński 1994). Bei dieser Studie wurde allerdings leider nicht nach Geschlecht unterschieden. 
diesem Fall bedeutet die Migration die Bewegung zwischen zwei unterschiedlichen kulturellen Systemen der Privatsphäre, in denen die Frauen unterschiedliche Stellungen einnehmen müssen. Die kulturellen Muster und gesellschaftlichen Rollen in diesen Sphären haben im Leben der Migrantinnen verschiedene Funktionen. Sie sind das kulturelle Kapital, nicht nur in Form der Qualifikationen zur Hausarbeit, sondern auch als Mittel zur Interpretation der eigenen Lage zwischen den Arbeitgebern und Schützlingen und der Vermittlung zwischen den beiden Bereichen der Privatsphäre.

\section{Pendelmigrantinnen aus Polen}

Die Grundlage der Analyse der Handlungen der Migrantinnen in der privaten Sphäre bilden 20 semi-strukturierte Interviews, die mit Migrantinnen durchgeführt wurden, die entweder als Pfegerinnnen älterer oder behinderter Personen oder als Reinigungskräfte in Haushalten arbeiten.

Die Auswahl der Stichprobe war zum größten Teil bedingt durch die Tatsache, dass die im Ausland arbeitenden Frauen meistens illegal beschäftigt werden, weshalb sie nur ungern einem Interview zustimmen. Aus ähnlichen Gründen - dem Gefühl der Illegalität und, in manchen Fällen, weil sie gleichzeitig Sozialhilfe beziehen oder in Polen arbeitslos gemeldet sind - entscheiden sich die MigrantInnen und ihre Familien, die eigene Migration nicht zuzugeben. In der Folge erlaubten uns die MigrantInnen oft nicht, das Gespräch aufzunehmen. Ferner beeinflusste der niedrige soziale Status der im Ausland ausgeübten Tätigkeit die endgültige Form des von uns gesammelten Materials. Das betrifft vor allem die Frauen, die als Reinigungskraft eingesetzt werden ${ }^{6}$, weil ihre Arbeit - anders als bei Haushälterinnen und Kinderbetreuerinnen - als soziale Degradierung und Marginalisierung in der Aufnahmegesellschaft betrachtet wird. Sowohl die negativen Meinungen als auch der gegenwärtige Diskurs über die negativen Folgen der Frauenmigration auf ihre Familien schränken die Wahl der Autopräsentationsstrategien der MigrantInnen in einem Interview ${ }^{7}$ ein und beeinflussen letztendlich den Verlauf der Interviews.

Die interviewten Migrantinnen bilden eine nach Alter, Ausbildung und familiärer Situation differenzierte Gruppe, die sich von der Sozialstruktur

6 Der niedrige Status dieses Berufs spiegelt sich in der Sprache wider: „Putzfrau“ hat in der polnischen Sprache eine negative und abwertende Konnotation, weshalb ich im Text Begriffe wie „Reinigungskraft" benutze.

7 Eine der Interviewpartnerinnen - die laut der Informationen einer mit ihr verwandten Vermittlerin unter anderen im Haushalt der Arbeitgebern geputzt hat fokussierte sich in dem Interview auf ihre spätere Karriere im Postamt in Polen und sprach kein Wort über das Putzen. 
Polens und der Struktur der Migrantinnen unterscheidet. In Bezug auf den Zeitpunkt ihres Lebens, an dem die Migrantinnen die Entscheidung über Auswanderung trafen, dominieren ältere Frauen. Acht der interviewten Frauen $(40 \%)$ waren bei der ersten Auswanderung über 44 Jahre alt, darunter 6 (30\%) sogar über 50. Im Vergleich zu den Schätzungen, dass die Migrantinnen über 50 19\% aller Migranten bilden (Kępińska 2003), ist diese Gruppe bei unserer Probe überrepräsentiert. Laut derselben Schätzung, dass 63.8\% der Migrantinnen zwischen 20 und 49 Jahre alt sind, wenn - bezüglich des Lebenszyklus - wichtige Veränderungen im Privat- und Berufsleben der Frauen auftreten. Neun (45\%) unserer Probandinnen waren zum Zeitpunkt der Migration jünger als 44.

Tabelle 1: Altersstruktur der Migrantinnen nach Lebensphasen

\begin{tabular}{|l|c|c|}
\hline Lebensphase & $\begin{array}{l}\text { Alter der Frauen zum Zeitpunkt } \\
\text { der ersten Migration }\end{array}$ & $\begin{array}{l}\text { Alter der Frauen zum Zeitpunkt } \\
\text { des Interviews }\end{array}$ \\
\hline unter 24 & $3(15 \%)$ & - \\
\hline $25-34$ & $5(25 \%)$ & $3(15 \%)$ \\
\hline $35-44$ & $4(20 \%)$ & $6(30 \%)$ \\
\hline $45-59$ & $8(40 \%)$ & $10(50 \%)$ \\
\hline über 60 & - & $1(5 \%)$ \\
\hline
\end{tabular}

Im Bezug auf den sozialen Status und die Struktur der Familie unterschieden sich die Probandinnen sowohl unter einander als auch von Migrantinnen allgemein. Die zahlreichste Gruppe bilden ledige und allein stehende Migrantinnen (56.7\%); 42.5\% der Frauen sind verheiratet (Kępińska 2003). Unter unseren Probandinnen ist die Struktur umgekehrt - 11 von ihnen sind verheiratet, 5 ledig und 4 geschieden.

Tabelle 2: Die Struktur der untersuchten Gruppe bezüglich des Familienstands und der Struktur des Haushalts

\begin{tabular}{|l|c|c|c|c|c|c|c|c|}
\hline \multirow{2}{*}{ Familienstand } & \multirow{2}{*}{ Single-Haushalt } & \multicolumn{9}{|c|}{$\begin{array}{l}\text { Ehemann, Kinder } \\
\text { (nach Zahl der Kindern) }\end{array}$} & Drei Generationen \\
\hline & & 0 & 1 & 2 & 3 & 4 & 5 & \\
\hline Ledig & 2 & & & 1 & & & & 1 \\
\hline Verheiratet & & 2 & 1 & 3 & 1 & 1 & 2 & 1 \\
\hline Geschieden & 2 & & 1 & 1 & & & & \\
\hline Gesamt & 4 & \multicolumn{7}{|c|}{13} \\
\hline
\end{tabular}

Die in der Tabelle dargestellten Daten charakterisieren die Differenzierung der Haushaltsgröße und -Modelle. In 8 Fällen haben wir es mit einer Kernfa- 
milien zu tun (Migrantin, ihr Ehemann und zumindest ein Kind, wobei in über der Hälfte der Fälle zur Familie 3 oder mehr Kinder gehören - meistens von über 45-jährigen Migrantinnen). Charakteristisch ist, dass häufig die erwachsenen Kinder bei den Eltern wohnen. Zum Zeitpunkt der Migration kommen sechs Migrantinnen für zumindest ein Kind über 12 Jahre auf. Zwei Haushalte bestehen aus nur der Frau und ihrem Ehemann. Zwei Respondinnen gehören zu Drei-Generationen-Haushalten: einmal mit Migrantin, ihren Eltern und einer Großtante, einmal mit Migrantin, Ehemann und Kind und Schwiegermutter.

Die unterschiedliche Haushaltsstruktur der Migrantinnen reflektiert die sich dynamisch wandelnde demographische Struktur der polnischen Gesellschaft (Kotowska 2005). ${ }^{8}$ Im Kontrast zu den Präferenzstrukturen ${ }^{9}$ ist, laut Nationalem Zensus 2002, in 40\% der Familien die Frau das Familienoberhaupt $^{10}$, was auf ein partnerschaftliches Modell der Familie hindeutet, in dem beide Ehepartner berufstätig sind und der Mann sich an der Kindererziehung und -betreuung beteiligt. Der Widerspruch zwischen dem gewünschten und realisierten Modell wurde zum großen Teil durch die Systemtransformation nach 1989 und den Wandel des meist traditionellen, wenn nicht konservativen Wertesystems verursacht. ${ }^{11}$

Die Ausbildungsstruktur der Stichprobe reflektiert die für die polnischen Migrantinnen typische Regel, dass vor allem Frauen mit sekundärer und Hochschulausbildung migrieren. Von 20 Frauen haben zumindest 15 eine se-

8 Die Präferenzen bezüglich des Familienmodells, das bei einer niedrigen Zahl von Ehescheidungen und Lebenspartnerschaften dominiert, bleiben jedoch unverändert. Laut dem Nationalen Zensus von 2002 bilden Verheiratete 55.6\% der Frauen, Geschiedene (oder getrennt Lebende) dagegen nur 5\%.

9 Den soziologischen Studien nach wird in der polnischen Gesellschaft ein patriarchalisches Familienmodell bevorzugt und als optimal empfunden, in dem Frauen und Männern ihre Rollen als Haushälterin und Ernährer klar zugewiesen sind (Cichomski/Jerzyński/Zieliński 1994). Ein ,umgekehrtes“ Modell, in dem eine Frau die Familie finanziell versorgt und ein Mann sich um die Kinder kümmert, wird als negativ und ungewollt von 94\% der Befragten gesehen (Rogulska 2006).

10 Im Mikrozensus wird das „Familienoberhaupt“ als die Person definiert, die vollständig oder zum größten Teil die Familie finanziell versorgt. Wenn mehrere Personen diese Rolle gleichermaßen erfüllen, dann ist das Familienoberhaupt die Person, die die finanziellen Mittel verwaltet (GUS 2003a). Ein Partnerschaftsmodell wurde nicht berücksichtigt.

11 Der Konservatismus ist in diesem Fall mit der unvollendeten Modernisierung und dem mangelnden Wandel der Sozialstruktur verbunden. Nicht ohne Bedeutung ist der tief greifende polnische Katholizismus. Die Katholische Kirche wurde im Sozialismus sogar gestärkt und nach 1989 gewann sie in der politischen und öffentlichen Sphäre volle Legitimation. Ihre wichtige Stellung bestätigen die Zahlen zur Taufe und zur Beteilung an Sonntagsmessen. 2004 waren 96\% der Bevölkerung katholisch getauft, davon nehmen $43 \%$ regelmäßig an Sonntagsmessen teil (http://www.iskk.ecclesia.org.pl/praktyki-niedzielne.htm). 
kundäre Ausbildung abgeschlossen und sechs von ihnen haben sich nach der Schule weitergebildet. Bezüglich der Berufsgruppen dominieren typisch weibliche und intellektuelle Berufe. Die Berufsstruktur der Migrantinnen wurde aufgrund von Angaben der Informantinnen bezüglich ihrer Beschäftigung rekonstruiert. Deswegen die in der Tabelle 3 dargestellten Berufe beziehen sich sowohl auf die Vergangenheit (im Fall von Frauen, die während des Aufenthalts in Deutschland ein anderes Beruf ausüben, als sonst in Polen, oder, im Fall von Rentnerin, auf das vorher ausgeübte Beschäftigung) als auch auf die Gegenwart (im Fall von Krankenschwestern, die sowohl in Poland als auch in Deutschland diesen Beruf ausüben).

\section{Tabelle 3: Berufsstruktur der Migrantinnen}

\begin{tabular}{|l|c|}
\hline Ausgeübter Beruf & Zahl der Migrantinnen \\
\hline Verwaltung und Büroarbeit & 2 \\
\hline Lehre & 2 \\
\hline $\begin{array}{l}\text { Dienstleistungen } \\
\text { (Handel und Gastronomie) }\end{array}$ & 6 \\
\hline Industrie & 5 \\
\hline Krankenschwester & 2 \\
\hline Eigene Firma & 1 \\
\hline Reinigungskraft & 1 \\
\hline War nie berufstätig & 1 \\
\hline
\end{tabular}

Die Migrantinnen haben ihren beruflichen Status nicht angegeben, man kann jedoch vermuten, dass die für die Industrie ausgebildeten Frauen zuletzt arbeitslos waren. In dieser Gruppe dominieren Frauen, die sich für die Migration erst nach dem 50. Geburtstag entschieden haben. Daraus kann man vermuten, dass sie entlassen oder in ein Frühpensionierungsprogramm aufgenommen wurden, welches, im Rahmen der Restrukturierung der polnischen Industrie, ein Versuch der Minimierung der sozialen Kosten der Beschäftigungsreduktion war. Die interviewten Frauen haben sich nicht als arbeitslose Personen definiert, was der patriarchalischen Geschlechterordnung entspricht, in der die Männer diesen Status bekommen, während die Frauen als ,nicht berufstätig“ oder „Hausfrau“ bezeichnet werden.

In Bezug auf ihren Herkunftsort überwiegen die Respondinnen (7) aus Regionen mit ethnischer Migrationstradition - Oberschlesien und Oppeln (Grabowska-Lusińska 2005). Auch Frauen aus Kleinpolen (10) sind relativ gut vertreten, einer Region mit gut ausgebauten familiären und freundschaftlichen Netzwerken ins Ausland und einer relativ niedrigen und territorial begrenzten Binnenmobilität. Die Stichprobe bilden vor allem Frauen (10) aus Städten mit über 100.000 Einwohnern, weitere sechs Migrantinnen kommen aus kleinen Städten (10.000-30.000 Einwohner) und vier wohnten in kleinen 
Ortschaften in der Nähe von größeren Städten. Während unserer Suche nach Informantinnen stellte sich heraus, dass Frauen in der ländlichen Bevölkerung in Südpolen die Migration nach Österreich und neuerdings auch nach Italien wählen (vgl. Slany/Małek 2005).

Zwei Typen von Migrantinnen zeichnen sich in der untersuchten Gruppe ab. Einen bilden die Frauen, die bereits in den 1990ern, in der Zeit der zunehmenden Migrationsaktivität von sozial marginalisierten Gruppen (Okólski 2002), nach Deutschland migrierten. In dieser Gruppe finden wir vor allem Frauen mit primärer und beruflicher Ausbildung, langjährige Pendelmigrantinnen oder Frauen mit einer von langen Unterbrechungen gekennzeichneten Migrationskarriere. Den zweiten Typus bilden Frauen, die nach dem Beitritt Polens in die EU ihre erste Migrationserfahrung hatten und sich in der ersten Phase der Migration befinden, in der sie nach dem ersten Aufenthalt im Ausland eine Bilanz der Vorteile und Nachteile ihrer Migrationsentscheidung machen.

In der untersuchten Gruppe nur zwei der Informantinnen waren über ein Jahr lang nicht mehr in Deutschland und planen keinen weiteren Aufenthalt dort in der nächsten Zeit. Der durchschnittliche Pendelrhythmus (Arbeit in Deutschland-Aufenthalt in Polen) betraf zwei bis drei Monate (14 Frauen). In den weiteren Fällen haben wir mit drei Typen zu tun: 1) selten und unregelmäßig reisende nach Polen (in Abständen von 2-8 Monaten - 2 Frauen), 2) unregelmäßig und selten reisende nach Deutschland (für 1-2 Monate - 2 Frauen), 3) selten reisende nach Polen, die in Deutschland dauerhaft leben wollen.

Die meisten der Frauen nutzten die Migrationsnetzwerke, überwiegend die Familienmitglieder, die bereits temporär oder dauerhaft in Deutschland arbeiteten. Seltener haben sich die Informantinnen auf nachbarschaftliche Beziehungen oder Arbeitskolleginnen verlassen. Nur zwei Frauen gaben an, selbständig und ohne Hilfe Arbeit gefunden zu haben.

Die meisten interviewten Migrantinnen arbeiteten in Deutschland als Haushälterinnen und Pflegerinnen für ältere und/oder behinderte Personen. Nur 5 von ihnen reinigten private Gebäude, wobei nur drei von ihnen diese Arbeit als ihre Hauptbeschäftigung in Deutschland bezeichneten. Nur einer der als Haushälterinnen beschäftigten Frauen wohnte außerhalb ihres Arbeitsplatzes. Eine der Pflegerinnen arbeitete zusätzlich als Reinigungskraft in einem anderen privaten Haushalt und als Kellnerin in einem Restaurant. Die Struktur der Stichprobe bietet uns die Möglichkeit, die unterschiedlichen Einstellungen der interviewten Frauen zur ausgeübten Arbeit zu analysieren und liefert uns interessantes Material für die Untersuchung von Effekten des $\mathrm{Zu}$ sammenlebens mit den Arbeitgebern. 


\section{Das Selbstbild der Migrantinnen}

Die interne Ausdifferenzierung der Stichprobe eignet sich besonders für die qualitative Analyse der Fallstudien. Diese Methode erlaubt, die von feministischen Autorinnen postulierte Subjektivität in Bezug auf die untersuchten Personen zu bewahren, hilft, Generalisierungen zu vermeiden und die Perspektive der Interviewpartnerinnen auf der Ebene der Interpretation eigener Erfahrungen mit einzubeziehen (Reinhard 1992).

Die Migrantinnen bilden ihre Erzählungen um zwei Motive herum - die unmittelbare Nähe zur Familie und die Nennung der sozialen und kulturellen Distanz zwischen Polen und Deutschland. Das erste Motiv verweist auf den heimatlichen kulturellen Raum, vor allem auf die soziale Rolle der Interviewpartnerin in Polen; das zweite richtete die Aufmerksamkeit auf den Aufenthalt im Ausland. Beide Perspektiven sind in den Interviews präsent, wobei die erste dominiert. Nicht ohne Bedeutung für die Form der Selbstpräsentationen der Migrantinnen war die Tatsache, dass der Interviewer/die Interviewerin keine Migrationserfahrung hatte.

Die meisten Interviewpartnerinnen präsentierten ihre Erfahrung mit Arbeit im Ausland als Erfolgstory. Die Erzählungen beinhalten mehrere Geschichten, mit deren Hilfe die Migrantinnen eine mehrdimensionale und positive Bilanz der eigenen Erfahrung herstellen wollen. Diese Erfolgsstories haben ein ähnliches Szenario, das folgende Elemente bilden: eine nicht unbedingt chronologisch präsentierte erste Darstellung des Problems, die Vorstellung der von der Frau als Reaktion darauf unternommenen Handlungen und ein Ergebnis dessen, das als Überwindung der Ausgangsprobleme beschrieben wird.

Dieses Szenario nutzen die Migrantinnen in Bezug auf drei Stränge der Migrationsbiographie: berufliche Selbsterfüllung im Rahmen der Arbeit im Ausland, Prozess der Integration in das deutsche Umfeld und die Organisation des Familienlebens und Haushalts in Polen während der Abwesenheit der Migrantin. Die Migrantinnen unterstreichen, dass es ihnen persönlich und ihren Familien gelungen war, die Schwierigkeiten zu überwinden und die mit der Migration verbundenen Risiken zu vermeiden. Dabei widersprechen sie oft den generalisierenden Meinungen über die berufliche und soziale Degradierung der Migranten und ihre Marginalisierung am Arbeitsort und Pathologisierung der Familien von Migranten.

Die Konstruktion der Erfolgsgeschichte im Kontrast zum Migrationsdiskurs verweist auch auf die Tendenz zur Selbstdarstellung in Einklang mit den in Polen dominierenden kulturellen Leitbildern des Geschlechts, die - in der Selbstdarstellung der Migrantinnen - weder zur Debatte stehen noch für die Zeit im Ausland aufgehoben werden können. Deswegen spielen die Aspekte der Sitte, vor allem die Folge dessen, dass die Migrantinnen sich für einige 
Zeit außerhalb der sozialen Kontrolle des Herkunftsumfelds aufhalten, in den Interviews nur eine marginale Rolle und werden gelegentlich in die Geschichte eingebaut, um die eigene „Treue“ zu den polnischen Normen zu demonstrieren. Diesem Ziel dienen auch die Gerüchte über die „Degeneration“ mancher Migrantinnen, die von den Informantinnen als „Mafia“ bezeichnet wurden. $\mathrm{Zu}$ dieser Gruppe gehören erfahrene Migrantinnen, die als Vermittlerinnen zwischen den Arbeitssuchenden aus Polen und den Deutschen arbeiten, die aber die Polinnen angeblich zu kriminellem Verhalten und sexuellen Praktiken mit den Arbeitgebern zwingen.

In den Vordergrund rückten dafür die mit der sozialen Rolle verbundenen Aspekte $^{12}$, wobei die Interviewpartnerinnen vor allem die Tatsache betonten, dass Arbeits-Auslandsmigrationen eine Verpflichtung gegenüber den Familien und primär den Kindern sind und dass eine lange Abwesenheit der Frau in Polen keine negativen Auswirkungen auf das Funktionieren der Familie hat. Die Darstellung der Migration in den Kategorien der Aufopferung der Frau ist ein Mittel zur Legitimierung der Migration. Aus dieser Perspektive ist Migration eine durch die Lebenssituation erzwungene Form der Erfüllung traditioneller Verpflichtungen, von denen im Vordergrund die Sorge für den Nachwuchs und den Haushalt steht. Diese Interpretationsstrategie ist nicht nur eine polnische Eigenschaft, sondern tritt oft in den Ländern auf, die von einer traditionellen (patriarchalischen) sozialen Ordnung und einem gleichzeitigen hohen Anteil an Frauen in den Migrationbewegungen charakterisiert sind, zum Beispiel in Asien oder Lateinamerika (Grasmuck/Pessar 1991; De Jong/ Richter/Isarabhakdi 1996; Tacoli 1999).

\section{Häusliches Matriarchat}

In der Darstellung der Arbeit in Deutschland beziehen sich die Migrantinnen gern und häufig auf familien- und haushaltsbezogene Kategorien, die zur heimatlichen kulturellen Sphäre gehören (Lawson 1998). Die „Privatisierung“ der Erzählung ist nicht nur ein Ergebnis der Beschäftigung im häuslichen Bereich, sondern auch des Mangels an möglichen Mustern im klassischen Repertoire der Migrationsbeziehungen, mit deren Hilfe die Frauen auf eine erfüllende und adäquate Art ihre eigenen Erfahrungen beschreiben könnten. In

12 Laut der von Mikuta (2000) durchgeführten Befragung üben die Frauen in Polen fast alle häuslichen Tätigkeiten aus, außer Reparaturen von elektrischen Haushaltsgeräten und Renovierungen. Als ausschließlich ihre Verpflichtungen haben die Frauen Personenpflege, Reinigung, Wäsche und Kochen genannt. Berufstätige Frauen sind durchschnittlich 4.3 Stunden mit Hausarbeit beschäftigt, Hausfrauen 8.6 Stunden. Gleichzeitig deklarieren aber die meisten Frauen, dass sie ein partnerschaftliches Ehemodell bevorzugen. 
dieser Situation nutzten die Interviewpartnerinnen gern die Elemente, die das so genannte häusliche Matriarchat-System von Normen und Praktiken bildeten. Walczewska bemerkte bei der Analyse des heutigen Emanzipationsdiskurses und der Geschlechterordnung in Polen, dass die Marginalisierung oder Ausschließung der Frauen in der Öffentlichkeit von der Dominanz der Mutter in der privaten Sphäre begleitet wird, der Mutter, die eine reale Macht über die Familie und den von ihr geführten Haushalt hat. Das häusliche Matriarchat wird auf mehreren Ebenen realisiert:

- Organisation und Management der Arbeiten im Haushalt,

- Kontrolle über die Kinder und ihre Sozialisation in Übereinstimmung mit dem traditionellen kulturellen Muster,

- ökonomische Entscheidungen bezüglich der privaten Sphäre.

Das häusliche Matriarchat ist teilweise ein Ergebnis der kulturell bedingten Überzeugung, dass die Frau eine entscheidende Rolle für das gelungene Funktionieren einer Familie hat, weil sie eine ,angeborene“ Fähigkeit zur Haushaltsführung sowie ein ,angeborenes“ Verantwortungsbewusstsein für die Familie besitzt. Die beiden sozialen Rollen der Hausfrau und Mutter spiegeln sich in alltäglichen häuslichen Aktivitäten wider, was Brach-Czaina (1998) das „Herumgetue“ nennt. Im häuslich-familiären Raum verwischt die Grenze zwischen Arbeitszeit und Freizeit. Dabei wird die geschlechtliche Arbeitsteilung beibehalten und die Tätigkeiten, die nicht mit dem Haushalt und der Familie verbunden sind, abgewertet, in Folge dessen die Aktivität der Frauen in der öffentlichen Sphäre (z.B. Berufstätigkeit) als eine gewisse Anomalie oder ein Störfaktor für die Familie gesehen. Die Frauen betrachten Berufstätigkeit in den Kategorien zusätzlicher Aktivität für das Wohlbefinden der Familie und nicht als eine Erfüllung eigener Aspirationen, wobei sie den eigenen finanziellen Beitrag zum Familienbudget marginalisieren.

Eine immanente Eigenschaft des häuslichen Matriarchatsystems ist seine Unterordnung unter die patriarchalischen Regeln, die auch in der Privatsphäre gelten. Auf der Ebene der kulturellen Normen behält der Mann seine Rolle als Hauptverdiener für die Familie, wobei dieser Status vor allem in der öffentlichen Sphäre und durch Rituale und symbolische Handlungen realisiert wird, was die Marginalisierung im häuslichen Bereich ausgleicht (Delumeau/Roche 1995).

Das häusliche Matriarchatsystem findet sich in den Erzählungen der Frauen wider, die seine Elemente bei der Darstellung der Beziehungen innerhalb der eigenen Familie und bei der Interpretation des Aufenthalts in Deutschland einbeziehen. Das matriarchalische „Drehbuch“ erlaubt ihnen, beide Phasen des Migranten-Daseins zu beschreiben, gleicht die Unterscheide in ihrem sozialen Status in Polen und in Deutschland aus und vereinfacht das Beibehalten der Geschlechtsidentität, die um Mutterschaft und Haushaltsführung konstru- 
iert wird. Ein gutes Beispiel der Erzählungskontinuität um ein häusliches Matriarchat ist die Aussage von Dorota Szafraniec, die sich bei der Darstellung der eigenen Stellung am Arbeitsplatz auf den breiteren Kontext der von ihnen ausgeübten Machtpraktiken bezieht, die ein Bestandteil ihrer Identität sind: „Ich glaube, ich habe überall Macht. Wenn aber für mich möglich, dann sehr unauffällig“.

\section{Quasi-Familie}

Auf der sprachlichen und inhaltlichen Ebene der Erzählungen verwischen die Migrantinnen die Unterschiede zwischen der eigenen Familie und des eigenen Haushalts und dem Arbeitsplatz. Bei der Beschreibung der Arbeitsbedingungen und, noch deutlicher, bei der Darstellung der Beziehungen zwischen sich selbst und den Arbeitgebern und Schützlingen, beziehen sich die Migrantinnen auf Kategorien, die sie auch dem eigenen häuslichen Raum in Polen zuschreiben. Alle Frauen, die als Pflegerinnen arbeiten und normalerweise von den Verwandten des Pflegebedürftigen eingestellt werden, definieren die Deutschen durch ihre Stellung in den familiären Beziehungsnetzen. Sie werden als Tochter, Sohn, die Cousine oder Ehefrau und die Pflegebedürftigen als Oma oder Opa bezeichnet. Diese letzte Gewohnheit, die älteren Menschen Oma oder Opa zu nennen, ist nicht nur eine Form der Identifikation nach dem Alter, verweist aber auch auf ein weiteres Muster, in dem der Schützling, der alleine nicht leben kann, kontrolliert und den Entscheidungen der um eine oder zwei Generationen jüngeren Pflegerin untergeordnet ist, die ihre Bestimmungen direkt mit ihrem Arbeitgeber bespricht. Typisch für diese Strategie ist das folgende Zitat aus dem Interview mit Roma Werner (29 Jahre alt), die seit Juli 2001 als Pflegerin der älteren, behinderten Mutter ihres Arbeitgebers beschäftigt ist:

„Ich hatte vierundzwanzig zur Verfügung bei der Oma, weil sie ein lebender Mensch ist und sie braucht einfach rund um die Uhr Pflege. Es ist nicht so, dass sie in der Nacht aufwacht und ich nicht dabei bin. Ich muss einfach bei ihr sein, weil wenn sie Pipi machen will und alleine nicht auf die Toilette geht, sie sich nichts zum Essen nehmen kann, alleine kann sie sogar aus dem Bett nicht raus. Weil sie krank ist, ja? Ich hatte zwei Stunden Pause eigentlich für mich ich hatte mich einfach ausgeschaltet, ich habe die Oma vor den Fernseher gesetzt: „Oma du sitzt jetzt hier und störe mich nicht" oder ihre Tochter ist zu ihr gekommen und ich konnte raus spazieren gehen oder einkaufen [...]. Die Zeit hatten wir so aufgeteilt: nach dem Mittagessen ging Oma auf den Sessel vor dem Fernseher und ich hatte eine kurze Pause, eigentlich frei. Dann haben wir mit Oma einen Film geguckt [...] alles zusammen ein Stündchen oder eineinhalb haben wir vom Fernseher verbracht. Dann habe ich ihre Füße oder Hände massiert, so einfach. Ich musste mich um sie kümmern, mit ihr 
irgendwie inzwischen sprechen. Die ganze Zeit haben wir gesprochen, Witze erzählt, oder sie hat was gelesen. Um sechs Uhr war eine Zwischenmahlzeit, und zwischendurch die Pillen [...] viele Pillen hat sie genommen. Und das war auch meine Pflicht, diese Pillen vorzubereiten, und wenn sie fast alle waren, dann musste ich die Tochter informieren, dass sie die Pillen kaufen sollte. Und ich musste auch zusehen, dass die Oma diese Pillen einnimmt, und die Oma wollte es nicht immer. Zum Beispiel zum Trinken danach sollte man ihr ein Wasser geben, weil sie sie in den Tee rausgespuckt hat. Bei mir hat sie das einmal gemacht, aber bei meiner Freundin, die dort im Austausch war, hat sie das ein paar Mal gemacht und die Freundin hat bemerkt, dass die Pille im Tee schwamm oder auf dem Boden der Tasse lag. Dann haben wir einen Film geguckt, dann um halb zehn am Abend habe ich sie zum Schlafen vorbereitet. Als ich dort zum ersten Mal da war, dann ging Oma um Mitternacht, halb eins in der Nacht zum Schlafen und ich habe gesagt, o nein, ich muss morgen aufstehen und ich werde mit ihr nicht so lange sitzen ${ }^{e}$ und ich habe sie auf halb zehn umgestellt und so ging es auch. Und sie ist brav schlafen gegangen [Lachen].“

Roma hat vier Situationen in der Beziehung zu ihrem Schützling beschrieben. In der ersten wurde die Verfügbarkeit der Pflegerin nicht als ein Element ihrer beruflichen Verpflichtungen, sondern als ihres persönliches Engagement und ihre Empathie für die behinderte und pflegebedürftige Person dargestellt. Distanz zeigte sich erst in der nächsten Darstellung der Freizeitplanung. Roma hat hier volle Kontrolle über die vor dem Ferneseher sitzende unbewegliche Frau, deren mögliche Bedürfnisse marginalisiert sind. In der nächsten Beschreibung des gemeinsamen Nachmittags vor dem Fernseher rückt die Pflegearbeit (Massage) in den Hintergrund und die Erzählung über die informelle Beziehungen in der Form des freien Gespräches und der Interaktion nimmt ihren Platz ein. Die Szene des quasi-familiären Lebens wird mit dem Motiv der professionellen Kontrolle der Medizinannahme ergänzt. Die Beziehungen mit der Frau, obwohl in jeder Situation die Entscheidungen und die Kontrolle bei der Pflegerin liegen, sind sehr ausdifferenziert. Die eigene Stellung am Arbeitsplatz interpretiert Roma in jeder der Darstellungen als eine Mischung der drei gegensätzlichen Kategorien:

- Emotionales Engagement und berufliche Verpflichtungen

- Emotionale Distanz und Freizeit

- Emotionales Engagement, berufliche Verpflichtungen und Freizeit

- Emotionale Distanz und berufliche Verpflichtungen

Roma begreift ihre Arbeit nicht nur in den beruflichen Kategorien, sondern als viel komplexere mehrdimensionale Beziehung mit dem Schützling, die sie nur dann entsprechend beschreiben kann, wenn sie sich auch auf die Kategorien aus der Privatsphäre bezieht. 
Wenn der Arbeitgeber ein Mann ist, stellen die Migrantinnen ihre Beziehungen am Arbeitsplatz etwas anders dar. Olga Ratajczak (53 Jahre alt), die von einem behinderten, allein lebenden Mann (50 Jahre alt) beschäftigt wurde, nutzt in der Erzählung über ihn ausschließlich neutrale, in der Öffentlichkeit benutzte Formen „der Mann“ oder „Herr“. Ähnlich Teresa Zielińska (59 Jahre alt), beschäftigt von dem Ehemann einer behinderten Frau, nutzt in Bezug auf ihn den Ausdruck ,dieser Mann“. Der zur öffentlichen Sphäre gehörige Begriff „Herr“ passt nicht in die Erzählungen der Migrantinnen über die quasi-eheliche Partnerschaft, emotionales Engagement und die eigene dominierende Rolle im Haushalt des Arbeitgebers, der sich im Laufe der Erzählung als „Johannes“ darstellt, mit dem die Migrantin per „du“ ist. Diese Widersprüchlichkeit spiegelt die Probleme beider Interviewpartnerinnen in der Nutzung von Parallelen zum Eheleben wider, die sehr geeignet wären, sie bei der Darstellung der Wichtigkeit ihrer Stellung am Arbeitsplatz zu unterstützen, weil sie dort tatsächlich die Rolle der Hausfrau im Schema des häuslichen Matriarchats annehmen. „Herr“ dient den Migrantinnen als eine Form der Versicherung für den Fall, wenn jemand sie um unangebrachtes Benehmen und die Verletzung von Normen, die ihre Beziehung zu ihrem Arbeitgeber regulieren, verdächtigen sollte. In diesem Rahmen ist eine Erfolgsstory möglich, in der die Hauptrolle die Zufriedenheit mit der zentralen Stellung im Haushalt des Arbeitsgebers, Mitentscheidungsrecht und Autonomie bei der Organisation der häuslichen Verpflichtungen und vor allem das Gefühl, für den hilfslosen Mann unersetzbar zu sein, spielen. Die Antwort, die Olga auf die Frage nach den für sie wichtigsten Personen in Deutschland gegeben hat, illustriert diese Strategie am besten:

„Ich muss Ihnen sagen, mein Schützling, das ist ein Mann mit einem guten Herz. Ich kann nicht sagen, dass er ist...Wissen Sie, was für mich eigentlich schrecklich ist? Dass er weint, wenn ich wegfahre. Er ist den ganzen Tag traurig. Ich fahre immer sonntagabends weg, weil ich den Bus habe, und er ist den ganzen Sonntag so traurig, dass er nichts essen will obwohl er weiß, dass meine Wechselpartnerin kommt. Sie ist doch nicht so schlecht. Ich weiß nicht, ich weiß nicht, wenn sie weg fährt, weint er nicht. Und wenn ich weg fahre - weint er. Ich sage: , warum weinst du, ich komme doch zurück.' ,Nur ruf mich an, wenn du ankommst.',Gut.' Ich rufe immer an.“

\section{Machtspiel in der Privatsphäre}

Ob die Beziehungen am Arbeitsplatz die Form des häuslichen Matriarchats annehmen, hängt davon $a b$, ob der Arbeitgeber mit der Pflegerin und den Untergebenen zusammenwohnt. Wenn die Migrantin vom Ehepartner des Pflegebedürftigen beschäftigt wurden, ist der Wohnort der Migrantin und des 
Arbeitgebers gleich und die Machtbeziehungen in der Privatsphäre durch das Geschlecht des Arbeitgebers bestimmt. Wenn der Ehemann pflegebedürftig ist, interpretieren die Migrantinnen die dominierende Position der Ehefrau nicht in den Kategorien des Beschäftigungsverhältnisses, sondern mit der Brille des häuslichen Matriarchats. Diese Interpretation wird dadurch unterstützt, dass die deutsche Arbeitgeberin meistens zu Hause ist (pensioniert) und sich um den Haushalt kümmert. In dieser Situation definiert die Pflegerin - unabhängig von ihrem Alter - die eigene Stellung mit den Kategorien einer Tochter, die sich dem Willen der Mutter unterordnet und in Verpflichtungen eingeführt wird (Pflegedienst).

In einer solchen Situation befinden sich zwei Migrantinnen, Grażyna Makowska (54 Jahre alt) und Krystyna Olczak (52 Jahre alt), die sich abwechselnd um den behinderten Ehemann ihrer Arbeitgeberin kümmern, die sie aus Mangel an geeigneteren Begriffen „Oma“ nennen. Die Arbeitgeberin, obwohl bereits 78 Jahre alt, ist selbstständig, hat sich aber entschieden, die Polinnen für die schwereren häuslichen Arbeiten und die Pflege ihres Mannes zu beschäftigen. Die Beziehung zwischen den Migrantinnen und der Arbeitgeberin bestimmen einerseits Regeln, die für die Beziehungen am Arbeitsplatz charakteristisch sind und andererseits solche, die die private Sphäre ordnen. Zuerst führte diese Vermischung von Machtordnungen zu Konfliktsituationen und Missverständnissen, wobei deren Quelle nicht die Macht der Arbeitgeberin, sondern der Mangel an stabilen Grenzen zwischen den Ordnungen war, die von beiden Seiten unterschiedlich definiert wurden. Die heutigen Beziehungen zwischen den Migrantinnen und der Arbeitgeberin als auch die Verteilung der Verpflichtungen sind ein Ergebnis eines Prozesses der Anpassung an den Rhythmus der Arbeit und des Haushalts, der Verteilung der Aufgaben und des Rechts auf Freizeit:

„[Die Arbeitgeberin hat sich geändert] für besser, weil sie nicht mehr so bissig war. Wenn ich was machen möchte, dann mache ich das alleine und sie diktiert mir nicht, kontrolliert nicht. Klar, dass sie guckt, was ich mache, aber einfach ohne diese Bissigkeiten. Vorher waren das solche ,Unterschiebungen“, solche unsinnigen ,Unterschiebungen.' Das hat mich gestört, weil ich denke, dass wenn ich arbeiten gehe, dann um zu arbeiten und nicht um nichts zu tun und zu bescheißen, Entschuldigung [...] Dort gibt es kaum was zu tun [...] Oma bereitet Mittagessen bereits um zwölf vor. Zusammen sind das zwei Stunden für Aufräumen, stellen Sie sich vor, zumindest so ist mein Gefühl, dass ich diese zwei Stunden nichts zu tun habe und ich versuche, diese zwei Stunden oben zu sitzen so dass sie zumindest denkt, dass ich was tue obwohl ich nichts zu tun habe [...] Man ist sauer, weil man nichts zu tun hat und muss nur vorgeben, dass man was macht und sucht nach Arbeit [Lachen] Na, aber irgendwie schaffen wir es schon.“ 
Grażyna interpretiert die Praktiken der Arbeitgeberin und ihre eigene Stellung in dem Kontext der Regeln, die das Zusammenleben in der Privatsphäre regulieren, wobei dieses Verhalten - die Kontrolle, das Entscheiden über die Arbeit und den Rhythmus der Aufgaben - näher den Regeln ist, die eine Arbeitsbeziehung bestimmen. Später stimmt Grażyna der beruflichen Interpretation bezüglich ihrer Pflegedienste zu. Der Konflikt findet in den Situationen statt, die Grażyna als solche betrachtet, in denen ihre Fähigkeiten als Hausfrau in Frage gestellt werden oder die mit ihrem Konzept der guten Organisation der Hausarbeit nicht übereinstimmen und die ihr „Herumgetue“ verhindern, weil sie die Domäne der Arbeitgeberin sind:

„Die Oma steht vor sechs Uhr auf, schließt (dem Ehemann) die Sonde an, sie ist im Allgemeinen so ein Frühaufsteher, sie mag nicht lange schlafen. Na dann geht sie ins Bad, kommt runter, dort brüht sie den Kaffee, kauft die Brötchen ein, oder etwas noch dazu. Sie setzt sich, weil sie sehr viele Zeitungen liest, breitet die Zeitungen aus, macht das Frühstück. [...] Und so zehn, fünfzehn nach sieben muss ich runter kommen. Das ist so nicht fest gelegt, aber die Oma wartet schon und ich muss runter kommen, weil sie mit dem Frühstück wartet.“

In diesem Fall erwecken die von der Arbeitgeberin den Migrantinnen aufgedrungenen Rituale des Haushaltslebens und die sozialen Rollen keinen Widerspruch, weil sie mit dem bekannten Muster der Familienorganisation übereinstimmen und die Machtverhältnisse denen ähnlich sind, die die Migrantinnen aus dem eigenen Kulturkreis und der Privatsphäre kennen. Aus diesem Grund ist es nicht verwunderlich, dass die Arbeitgeberin einen Teil des Hauses alleine aufräumt oder dass sie kocht und einkauft - das sind die sichtbaren und akzeptierten Symbole der Macht einer Frau im eigenen Haushalt.

Die für das Funktionieren der Privatsphäre typischen Regeln stellten sich als sehr effektive Basis der Kommunikation in den Situationen heraus, die früher zu Konflikten führten. Beide Migrantinnen bemerkten die deutliche Veränderung der Interaktionen und der Weise, wie die Arbeitgeberin ihre Arbeit und sie betrachtet. Die Aufgaben selbst blieben unverändert, die Migrantinnen haben jedoch eine gewisse Freiheit in der Organisation ihrer Arbeit gewonnen.

„Ich räume genau das Wohnzimmer auf und wische den Staub und das reicht schon. Die Oma kümmert es nicht, nur dieses Zimmer muss sauber sein, und der Rest, das ist je nach meiner Idee. Das Putzen ist meine Sache, muss nur oft sein. Die Oma mischt sich nicht ein, sie steht dort nicht und kontrolliert nicht, was ich mache. Sie guckt irgendwohin, weil ich schon manchmal gesehen habe, als sie geguckt hat, ob irgendwo noch Staub liegt, aber Gott bewahre, sie geht nicht so offensichtlich hin, so wie manche, mit einem Tuch. So was ist mir nicht passiert. Ich funktioniere ein- 
fach wie, ich weiß nicht, wie ein Familienmitglied? Ich kann das sogar nicht nennen, klar, ich bin fremd, aber doch nicht so fremd."

„Ich war schockiert wie sich die Oma verändert hat, wie sich ihre Einstellung zu uns geändert hat. Aber ich denke, wir haben uns einfach an einander gewöhnt. Sie weiß schon, wer wir sind. Wir wissen, wer sie ist, wie wir uns an einander anpassen sollten. Und ich denke, dass es so ist. Dass wir uns einfach kennen lernen und dass es für uns so einfacher ist.“

Den endgültigen Erfolg kann man auch als das Ergebnis der pädagogischen Bemühungen der Arbeitsgeberin gegenüber den Migrantinnen verstehen. Vor allem die alltäglichen Anpassungen des Kommunikationsverhaltens und der Regeln des Zusammenlebens haben der Stabilisierung im Haushalt beigetragen. Diese wurde jedoch auf eine spezifische Weise erreicht, indem die Arbeitgeberin vermieden hat, ihre tatsächliche Macht den Migrantinnen zu demonstrieren (Bourdieu/Passeron 1990).

\section{Diskussion}

Die Analyse der Fälle, die hier präsentiert wurden, schöpft nicht alle Situationen und Erzählungen aus und beschränkt sich auf bestimmte Aspekte der Interpretationen der Arbeitssphäre mittels des eigenen, kulturell bestimmten Konzepts der Privatsphäre. Die quasi-familiäre Metaphorik zeigt sich vor allem in den Erzählungen der Migrantinnen, die um die Erfolgsstories konstruiert sind, in denen das Gefühl der Fremdheit ${ }^{13}$ im Migrationsumfeld und des sozialen und beruflichen Abstiegs marginalisiert wird. Migrantinnen stellen den Prozess der Integration im häuslichen Umfeld in Deutschland dar, dessen Beweis ist, dass sie emotionale, stabile und den Bereich der Arbeit überschreitende Bindungen entwickeln, sowie ein Kommunikationssystem am Arbeitsplatz, das sich auf die alltäglichen Rituale und das Verschwinden der Distanz zwischen Arbeitgebern und Arbeitnehmern stützt. Dieser Typ von Bindungen und Verhalten bezieht sich vor allem auf Migrantinnen, die als Pflegerinnen arbeiten und zusammen mit den Arbeitgebern (und Schützlingen) wohnen und deren Freizeit und Arbeitszeit integriert sind.

Trotz der Praktiken, die auf die Privatisierung der Arbeitssphäre in Deutschland gerichtet sind, überschreitet die Darstellung der Migrationserfahrung mehrmals die Begrifflichkeit aus der heimatlichen kulturellen Matrix.

13 Marginalisierung und Entfremdung erfolgen in den Erzählungen der Migrantinnen in den Situationen des Kontakts mit Bekannten und Verwandtschaft der Pflegebedürftigen und der Arbeitgeber, die den Migrantinnen der sozialen und kulturellen Distanz offenbaren. 
Das betrifft vor allem die Situationen, in denen die Ähnlichkeit beider Räume des Privaten nur scheinbar ist, wodurch die Beziehungen am Arbeitsplatz eine unerwünschte Bedeutung annehmen, die ein Brechen der ethischen und Verhaltensnormen suggeriert. Dann bildet die familiäre-häusliche Interpretation eine Barriere, die die Migrantinnen nicht durchbrechen können. ${ }^{14}$

$\mathrm{Zu}$ den negativen Effekten der Privatisierung kann man auch den Einfluss der Migrationserfahrung der Frauen auf die Festigung der konservativen Geschlechterordnung und ihr Prinzip der Zuschreibung von Frauen in die Privatsphäre zählen. Die Arbeit in Deutschland stützt sich auf die Praxis der Migrantinnen, die sie aus dem eigenen Kulturkreis kennen. Im Rahmen der Arbeit und des Lebenszyklus am Arbeitsplatz reproduzieren die Migrantinnen die Verhaltensmuster und die Normen, die mit den traditionellen pflegerischen und häuslichen Verpflichtungen einer Frau verbunden sind. Gleichzeitig, trotz des ökonomischen Gewinns aus der Arbeit in Deutschland, kommen die Migrantinnen nicht dazu, den Wert ihrer Arbeit im eigenen Haushalt zu reinterpretieren.

14 Ein weiterer negativer Aspekt der Privatisierung der Erfahrung ist das Aufgeben der Rechte des Arbeitnehmers, wie zum Beispiel Verwandeln über eine Gehaltserhöhung oder das Recht auf Freizeit. 
